\title{
Phonon engineering in nanostructures: Controlling interfacial thermal resistance in multilayer-graphene/dielectric heterojunctions
}

\author{
R. Mao, ${ }^{1}$ B. D. Kong, ${ }^{1}$ K. W. Kim, ${ }^{1}$ T. Jayasekera,${ }^{2}$ A. Calzolari, ${ }^{3, a)}$ \\ and M. Buongiorno Nardelli ${ }^{4, b)}$ \\ ${ }^{1}$ Department of Electrical and Computer Engineering, North Carolina State University, \\ Raleigh, North Carolina 27695-7911, USA \\ ${ }^{2}$ Department of Physics, Southern Illinois University, Carbondale, Illinois 62901, USA \\ ${ }^{3}$ Istituto Nanoscienze CNR-NANO-S3, I-41100 Modena, Italy \\ ${ }^{4}$ Department of Physics and Department of Chemistry, University of North Texas, Denton, Texas 76203, \\ USA and CSMD, Oak Ridge National Laboratory, Oak Ridge, Tennessee 37831, USA
}

(Received 2 July 2012; accepted 28 August 2012; published online 13 September 2012)

\begin{abstract}
Using calculations from first principles and the Landauer approach for phonon transport, we study the Kapitza resistance in selected multilayer graphene/dielectric heterojunctions (hexagonal BN and wurtzite $\mathrm{SiC})$ and demonstrate (i) the resistance variability $\left(\sim 50-700 \times 10^{-10} \mathrm{~m}^{2} \mathrm{~K} / \mathrm{W}\right)$ induced by vertical coupling, dimensionality, and atomistic structure of the system and (ii) the ability of understanding the intensity of the thermal transmittance in terms of the phonon distribution at the interface. Our results pave the way to the fundamental understanding of active phonon engineering by microscopic geometry design. (C) 2012 American Institute of Physics. [http://dx.doi.org/10.1063/1.4752437]
\end{abstract}

Recent progress in nanostructured materials synthesis and device fabrication technology has brought the need for thermal management to the forefront. For example, the power density needed to operate an integrated circuit chip is already nearing that of a nuclear reactor (around the $100 \mathrm{~W} / \mathrm{cm}^{2}$ mark) and could continue its climb with size scaling if no improvement is made. ${ }^{1}$ Effective strategies for heat transfer away from active devices and into heat sink regions need to be engineered in future nanometer-scale elements on heterogeneous material platforms for high integration density and performance. Hence, a fundamental understanding of thermal transport in the nanoscale environment is an essential requirement. In fact, the problem of thermal transport at the nanoscale inherently demands an atomistic, quantum-mechanical description of the complexity of the nanointerfaces.

Among the most promising materials for advanced microelectronic applications, graphene, a single plane or a few-layers of graphite, has taken center stage for its outstanding mechanical, electronic, and thermal properties. ${ }^{2-5}$ In particular, advances in the epitaxial growth of graphene films on dielectric substrates, such as hexagonal BN (h-BN) or $\mathrm{SiC}$, have the potential to open new classes of device applications that may revolutionize the semiconductor roadmap for future decades. ${ }^{6,7}$ Heat conduction between dielectrics can be interpreted as transport of phonons: quantized elastic waves of lattice vibrations. When the heat flows across interfaces, the scattering of the phonons originates a thermal resistance often known as Kapitza resistance. ${ }^{8}$ There are two classic models of evaluating the thermal resistance in materials: the acoustic mismatch and the diffuse mismatch model. ${ }^{9,10}$ Both are phenomenological approaches, assume linear phonon dispersions, oversimplify the effects from the

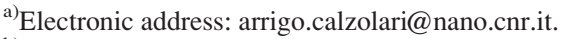

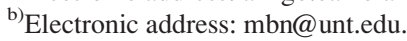

interfaces, and are fitted to reproduce existing experimental results. Beyond these simplified approaches, atomic level methods, such as molecular dynamics, ${ }^{11}$ lattice dynamics, ${ }^{12}$ and non equilibrium Greens functions, ${ }^{13,14}$ have been used to study thermal resistance in interfaces and superlattices. These methods have in common the need for interatomic potential functions for the evaluation of the dynamics of the systems for which only empirical models have been used to date. However, given the complexity of the interactions at nanoscale systems such as graphene/substrate interfaces and the impracticality of generating accurate potential functions for any specific case, first principles methods are clearly the ideal choice for these investigations.

In this letter, we have studied the heat conduction properties at the heterogeneous interface between multilayer graphene and selected dielectric substrate materials using a fully first principles approach for both the electronic and the thermal properties of the systems. In particular, we combine state-of-the-art density functional theory (DFT) and density functional perturbation theory (DFPT) ${ }^{15}$ calculations for the evaluation of the vibrational properties of nanoscale systems with the general framework of the Landauer approach ${ }^{16,17}$ for ballistic phonon transport using a real-space Green's function technique. ${ }^{13,14,18,19}$

At the nanoscale, thermal transport across a device connected to two thermal baths ( $L$ and $R$ ) can be described by extending the Landauer formalism for electrons to phonons. In this formalism, the expression for the thermal current density is

$$
J\left(T_{L}, T_{R}\right)=\frac{\hbar}{2 \pi} \int_{0}^{+\infty} d \omega \omega \mathcal{T}_{p h}(\omega)\left[n\left(T_{L}, \omega\right)-n\left(T_{R}, \omega\right)\right],
$$

where $n\left(T_{L, R}, \omega\right)$ are the Bose-Einstein distribution functions for the left or right lead, $T_{L, R}=T \pm \Delta T / 2$ are the temperatures 
of the thermal reservoirs, and $\mathcal{T}_{p h}(\omega)$ is the transmission function of the phonons. In the limit of small temperature difference $\Delta T$, the phonon thermal conductance $\kappa_{p h}(T)=J(T) / \Delta T$ is given by

$$
\kappa_{p h}(T)=\frac{1}{2 \pi \hbar} \int_{0}^{+\infty} d(\hbar \omega) \mathcal{T}_{p h}(\omega) \hbar \omega\left[\frac{\partial n(T, \omega)}{\partial T}\right] .
$$

The transmission functions $\mathcal{T}_{p h}(\omega)$ for phonons can be calculated using a real-space Green's function approach, similar to the one used for the electron transport across nanoscale interfaces, ${ }^{18}$ where the main ingredients are (i) the diagonal matrix $\mathbf{M}_{p h}$ corresponding to the masses of the atoms and (ii) the matrix $\mathbf{K}_{p h}(\mathbf{r})$ of the interatomic force constants (IFCs) in real space. ${ }^{20,21}$ The thermal resistance, $R(T)$, is then obtained by inverting Eq. (2) and normalizing to the interface area. In the present treatment, IFCs are calculated fully from first principles ${ }^{22}$ within DFPT ${ }^{15,23}$ that allows unequivocal consideration of the microscopic geometry as well as chemical and electronic modification at the interface without resorting to phenomenological or ad hoc models. For simplicity, we restrict our analysis only to the harmonic contribution to lattice dynamics. The inclusion of three phonon scattering effects, although obtainable from DFPT, ${ }^{15}$ is outside of the scope of this investigation. ${ }^{23}$

The device geometry considered for the evaluation of the interfacial thermal resistance is comprised of a heterostructure containing an interface between different materials connected to two thermal baths (reservoirs) at two different temperatures, $T_{L, R}$ (see Figure $\mathrm{S} 1$ in supplemental material $^{24}$ ). In this configuration and in the absence of inelastic scattering, the thermal resistance measured across the system, $R(T)$, coincides with the interfacial thermal resistance $R_{i n}(T)$, i.e., the Kapitza resistance through the graphene/ dielectric interface. For more details about the method see, for instance, Ref. 11 and references therein.

To understand thermal transport at multilayer graphene (G) and dielectric interfaces, we have considered two different classes of substrates, hexagonal $\mathrm{BN}$ and wurtzite $\mathrm{SiC}$. Both share a planar hexagonal symmetry in the direction perpendicular to the interface, but they have a different interlayer coupling in the direction parallel to transport: one is a 2-D layered (h-BN) and the other an intrinsically 3-D system (wurtzite $\mathrm{SiC}$ ). See Figures S2 and S3 in supplementary material ${ }^{24}$ for details on the geometry of the bulk and interface structures.

In the case of the h-BN substrate, the structure of the interface is planar without any buffer layer, so the model system for the transport calculation is a slab with eight h-BN planes and eight graphene layers. As for $\mathrm{SiC}$, we focus on multilayer graphene grown on the Si-face of $2 \mathrm{H}-\mathrm{SiC}(0001)$ $\left[\sqrt{3} \times \sqrt{3} \cos \left(30^{\circ}\right)\right]$ that is characterized by a carbon buffer layer bound to the $\mathrm{SiC}$ surface and an electron-doped graphene layer on top. More specifically, we consider both the native $(\mathrm{SiC})$ and the hydrogenated case $(\mathrm{H}: \mathrm{SiC})$, where $\mathrm{H}$ atoms passivate $\mathrm{Si}$ dangling bonds (lonely atoms) in the interface layer, ${ }^{25}$ to investigate how the coupling of the substrate with the graphene layers affects the phonon transport. In this case, the model system for the interface is comprised by a $\mathrm{SiC}$ slab (eight planes, $2 \times 2$ lateral periodicity), the
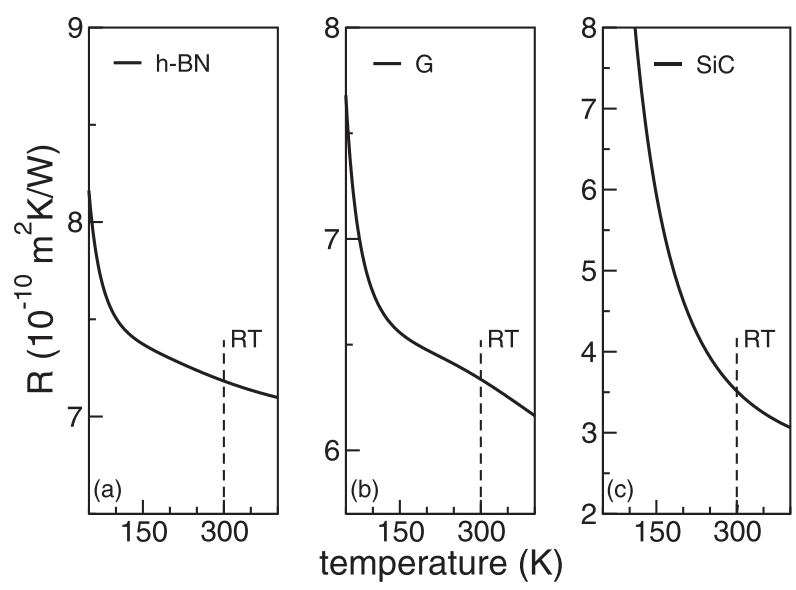

FIG. 1. The intrinsic contact thermal resistance for (a) h-BN, (b) G, and (c) $\mathrm{SiC}$ in the (0001) direction. Vertical dashed lines mark the $R$ values at room temperature.

carbon buffer layer (with or without hydrogenation), and three graphene layers ${ }^{26}$ (see Fig. S3 in supplementary material for details ${ }^{24}$ ).

We first discuss the characterization of the bulk systems and their intrinsic thermal contact resistance a la Landauer. The preliminary calculations of phonon dispersion and transmittance for the bulk systems can be found in the supplementary material. ${ }^{24}$ Using Eq. (2), we calculate the contact thermal resistance of the bulk h-BN, "bulk graphene" and bulk $\mathrm{SiC}$ as a function of temperature in the range 50-400 K, as shown in Fig. 1. The thermal resistances hold a $1 / \mathrm{T}$ dependence with room temperature values of $7.2 \times 10^{-10} \mathrm{~m}^{2} \mathrm{~K} / \mathrm{W}$ and $6.3 \times 10^{-10} \mathrm{~m}^{2} \mathrm{~K} / \mathrm{W}$ for the case of bulk h-BN and graphene, respectively. These numbers are consistent with the expectation of a small out-of-plane transmission in these layered systems, in good agreement with available experimental results. ${ }^{27,28}$ Similar results are found for bulk $\mathrm{SiC}$, with the important difference that now the contact resistance at room temperature is $3.5 \times 10^{-10} \mathrm{~m}^{2} \mathrm{~K} / \mathrm{W}$, i.e., almost half that of layered systems.

The same calculation route has been carried out for the whole interface structure of the $\mathrm{G} / \mathrm{h}-\mathrm{BN}$ system. The interfacial thermal resistances are plotted in Fig. 2. The relatively low (albeit still one order of magnitude larger than the bulk

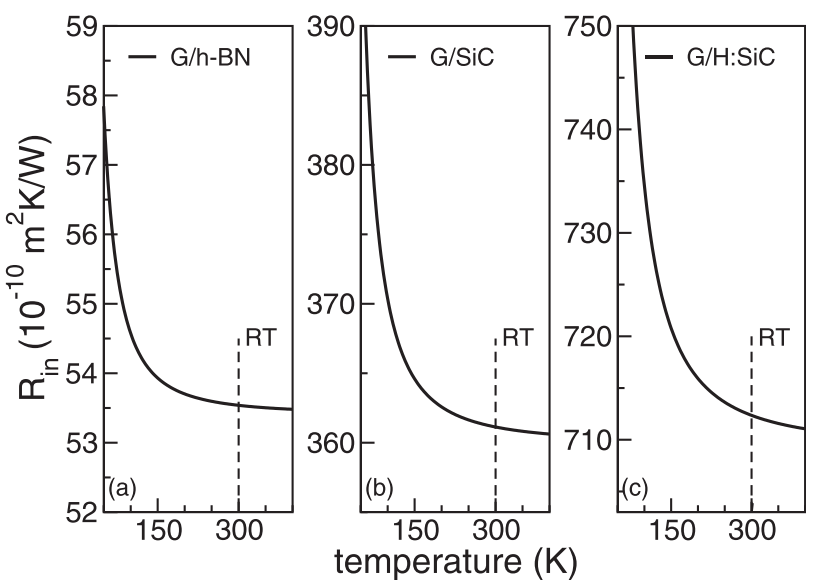

FIG. 2. The interfacial thermal resistance for (a) G/h-BN, (b) G/SiC, and (c) $\mathrm{G} / \mathrm{H}$ :SiC. Vertical dashed lines mark the $\mathrm{R}_{i n}$ values at room temperature. 
values) interfacial thermal resistance in this system indicates a sizable permeability of the system to heat transport and can be clearly understood in terms of the similarity of the phonon dispersions in the two sub-systems and the transferability of the force constants due to the similar masses of the $\mathrm{B}, \mathrm{N}$, and $\mathrm{C}$ atoms (mass approximation ${ }^{29}$ ). Our values are in good agreement with the experimentally observed thermal resistance of exfoliated (weakly coupled) graphene on $\mathrm{SiO}_{2}$ at room temperature $\left(56-120 \times 10^{-10} \mathrm{~m}^{2} \mathrm{~K} / \mathrm{W}\right){ }^{5}$ The interface indeed acts as a barrier to the transmission of phonons from one side of the device to the other.

The situation changes drastically when we consider interfaces with 3-D materials such as SiC. Looking at the results for the $\mathrm{G} / \mathrm{SiC}$ and $\mathrm{G} / \mathrm{H}: \mathrm{SiC}$ cases, shown in Figs. 2(b) and 2(c), it is clear that the thermal resistance depends critically on the microscopic details of the interfaces. For instance, the interfacial thermal resistance of the $\mathrm{SiC} / \mathrm{G}$ at $300 \mathrm{~K}$ is $361.1 \times 10^{-10} \mathrm{~m}^{2} \mathrm{~K} / \mathrm{W}$ one order of magnitude higher than in the G/h-BN case. This is due to the substantial difference between the phonon distribution functions in a 3-D bulk vs. a 2-D layered system. Even more drastic is the variation due to the microscopic modification of the interface by hydrogenation. Now the thermal resistance increases by a factor of $2\left(712.4 \times 10^{-10} \mathrm{~m}^{2} \mathrm{~K} / \mathrm{W}\right)$, indicating a strengthening of the interfacial barrier to phonon transmission.

In order to understand the thermal conductance features of these three systems, we have carried out a microscopic analysis of the transmittance in terms of the most relevant contribution from the vibrational normal modes at the interface. The results are summarized in Fig. 3 (left panel). A general feature, common to all the systems, is the suppression of the phonon modes for energies above $\sim 100 \mathrm{~cm}^{-1}$, which can be attributed to the lack of matching between the graphene layers and the substrate modes in the same energy region (see Figs. S4-S6 in supplementary material). ${ }^{24}$ Moreover, the individual analysis of the atomic displacements for the low energy modes ( $\$ 100 \mathrm{~cm}^{-1}$ ) that correspond to high transmittance values allows us to identify the general shape of the transport eigenchannels for the heat flux at those ener-

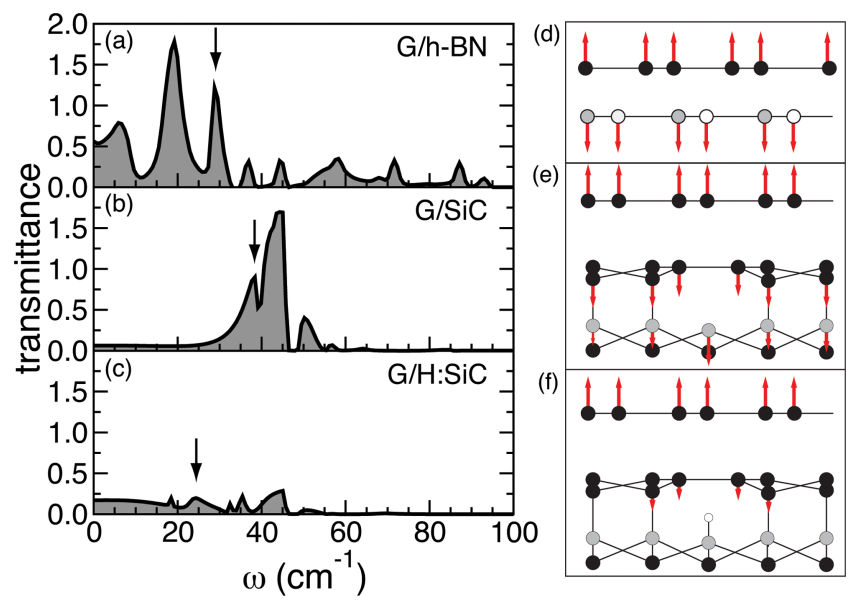

FIG. 3. Left panel: total transmittance for (a) G/h-BN, (b) G/SiC, and (c) $\mathrm{G} / \mathrm{H}: \mathrm{SiC}$. Right panel: sketch of the atomic displacement patterns of the relevant normal modes that are mainly responsible for the phonon transmission at the interface. The corresponding energies of modes (d), (e), and (f) are indicated by arrows in panels (a), (b), and (c), respectively. gies (Fig. 3, right panel). In the $\mathrm{G} / \mathrm{h}-\mathrm{BN}$ case, we find two representative sets of collective modes that are efficiently transmitted across the interface: at low frequencies (up to $\sim 25 \mathrm{~cm}^{-1}$ ), the modes have out-of-plane displacements as in Fig. 3(d).

In the intermediate energy range $\left(25-100 \mathrm{~cm}^{-1}\right)$, the modes have in-plane displacements (shear modes, parallel to the interface) and are more weakly coupled (hence the lower transmittance). In the SiC case [Fig. 3(b)], this transmission channel is inhibited by the 2-D to 3-D transition across the heterojunction. Now, only out-of-plane modes can transmit across the interface [Fig. 3(e)]. This effect is clearly a manifestation of the possibility of engineering phonon transport across an interface by the selective modification of the interface geometry. Indeed, this is further exemplified by the case of transmission through the $\mathrm{G} / \mathrm{H}: \mathrm{SiC}$ interface. The addition of a $\mathrm{H}$ atom on the $\mathrm{Si}$ lonely atom at the interface layer blocks the transmission of the out-of-plane mode responsible for most of the heat transfer around $\sim 40 \mathrm{~cm}^{-1}$. The $\mathrm{H}-\mathrm{Si}$ bond makes the interface rigid to out-of-plane modes and effectively blocks the transmission in the low energy range, as displayed by the displacement pattern of the corresponding mode (now red-shifted at $\sim 25 \mathrm{~cm}^{-1}$ ) and shown in Fig. 3(f). This is clearly interpretable in view of the breaking of the mass approximation by the insertion of a "mass defect," the light $\mathrm{H}$ atoms. Moreover, in addition to the mass effect, there is a slight variation of the interlayer distance between $\mathrm{G}$ and $\mathrm{SiC}$ upon $\mathrm{H}$ adsorption in the buffer layer $(3.89 \AA$ in SiC vs. $4.13 \AA$ in $\mathrm{H}: \mathrm{SiC})$, which induces a further weakening of the IFCs. Recent experiments have already shown that hydrogen functionalization can play an important role in manipulating thermal conductance in graphene-metal interfaces, ${ }^{30}$ an indirect confirmation of the validity, significance, and timeliness of our investigation.

These results point to the paramount importance of interfacial geometry for the control of thermal properties of a heterojunction: Indeed, interfaces can be engineered as to prevent or enhance heat transfer from one system to another and thus select the directionality of the heat dissipation channel. Depending on the application for any particular device, one should be able to design the desired phonon distribution function. For instance, in systems where one needs to capture thermal energy, e.g., solar thermal devices or thermoelectric systems, it would be desirable to collect the heat in the active layer, with negligible dissipation (as in $\mathrm{G} / \mathrm{H}: \mathrm{SiC}$ ). If instead heat dissipation is essential for maintaining high charge or spin carrier mobility in a device, a heterojunction like $\mathrm{G} / \mathrm{SiC}$ or G/h-BN would be the ideal choice. Finally, we would like to stress once again that this type of analysis is only possible because we are using a fully first principles approach where all the individual contributions are evaluated explicitly at a quantum mechanical level.

We would like to thank Yifeng Chen for his technical support and useful discussions. This work was supported, in part, by the DARPA/HRL CERA, SRC/NRI SWAN, and US ARO. M.B.N. wishes to acknowledge partial support from the Office of Basic Energy Sciences, U.S. Department of Energy at Oak Ridge National Laboratory under Contract No. DE-AC05-00OR22725 with UT-Battelle, LLC. Calculations 
have been run at NCCS-ORNL and HPC-NCSU. This work was supported in part by the SRC through Task ID P14924 in the Center for Electronic Materials Processing and Integration at the University of North Texas.

${ }^{1}$ E. Pop, S. Sinha, and K. E. Goodson, Proc. IEEE 94, 1587 (2006).

${ }^{2}$ A. K. Geim and K. S. Novoselov, Nature Mater. 6, 183 (2007).

${ }^{3}$ B. Kong, S. Paul, M. Buongiorno Nardelli, and K. Kim, Phys. Rev. B 80, 033406 (2009).

${ }^{4}$ W. W. Cai, A. L. Moore, Y. W. Zhu, X. S. Li, S. S. Chen, L. Shi, and R. S. Ruoff, Nano Lett. 10, 1645 (2010).

${ }^{5}$ Z. Chen, W. Jang, W. Bao, C. N. Lau, and C. Dames, Appl. Phys. Lett. 95, 161910 (2009).

${ }^{6}$ G. M. Rutter, J. N. Crain, N. P. Guisinger, T. Li, P. N. First, and J. A. Stroscio, Science 317, 219 (2007).

${ }^{7}$ C. R. Dean, A. F. Young, I. Meric, C. Lee, L. Wang, S. Sorgenfrei, K. Watanabe, T. Taniguchi, P. Kim, K. L. Shepard, and J. Hone, Nature Nanotechnol. 5, 722 (2010).

${ }^{8}$ G. Pollack, Rev. Mod. Phys. 41, 48 (1969).

${ }^{9}$ E. Swartz and R. Pohl, Rev. Mod. Phys. 61, 605 (1989).

${ }^{10}$ W. A. Little, Can. J. Phys. 37, 334 (1959).

${ }^{11}$ E. S. Landry and A. J. H. McGaughey, Phys. Rev. B 80, 165304 (2009).

${ }^{12}$ D. Young and H. Maris, Phys. Rev. B 40, 3685 (1989).

${ }^{13}$ W. Zhang, T. Fisher, and N. Mingo, Numer. Heat Transfer, Part B 51, 333 (2007).

${ }^{14}$ J. Wang, J. Wang, and J. Lü, Eur. Phys. J. B 62, 381 (2008).

${ }^{15}$ S. Baroni, S. De Gironcoli, A. Dal Corso, and P. Giannozzi, Rev. Mod. Phys. 73, 515 (2001).

${ }^{16}$ R. Landauer, IBM J. Res. Dev. 1, 223 (1957).

${ }^{17}$ Y. Imry and R. Landauer, Rev. Mod. Phys. 71, S306 (1999).

${ }^{18}$ M. Buongiorno Nardelli, Phys. Rev. B 60, 7828 (1999).

${ }^{19}$ S. Datta, Electronic Transport in Mesoscopic Systems (Cambridge University Press, Cambridge, 1995).
${ }^{20} \mathrm{X}$. Gonze and C. Lee, Phys. Rev. B 55, 10355 (1997).

${ }^{21}$ Y. Chen, T. Jayasekera, A. Calzolari, K. Kim, and M. Buongiorno Nardelli, J. Phys.: Condens. Matter 22, 372202 (2010).

${ }^{22}$ All the calculations are performed in the framework of DFT as it is implemented in QUANTUM-ESPRESSO package [P. Giannozzi, S. Baroni, N. Bonini, M. Calandra, R. Car, C. Cavazzoni, D. Ceresoli, G. L. Chiarotti, M. Cococcioni, I. Dabo et al., J. Phys.: Condens. Matter 21, 395502 (2009)]. We used LDA norm-conserving pseudopotentials for all the atomic species, a 65 Ry energy cut off in the plane wave expansion and a $12 \times 12 \times 2$ and $6 \times 6 \times 1$ regular k-point mesh for G/h-BN and G/SiC, respectively. Four q-points have been used in the direction of transport for the interfacial thermal resistance calculations.

${ }^{23}$ A. Calzolari, T. Jayasekera, K. W. Kim, and M. Buongiorno Nardelli, "Ab initio thermal transport properties of nanostructures from density functional perturbation theory" (unpublished).

${ }^{24}$ See supplementary material at http://dx.doi.org/10.1063/1.4752437 for schematic diagram of an interface between two semiinfinite leads (Fig. S1); for bulk and interface structures (Figs. S2 and S3); and for bulk BN, $\mathrm{G}$, and $\mathrm{SiC}$ characterization (Figs. S4-S6).

${ }^{25}$ T. Jayasekera, B. D. Kong, K. W. Kim, and M. Buongiorno Nardelli, Phys. Rev. Lett. 104, 146801 (2010).

${ }^{26}$ In all these geometries, there is a residual lattice mismatch between the graphene and the substrate to satisfy lateral periodic boundary conditions (graphene is isotropically strained $7 \%$ on $\mathrm{SiC}$ and $2 \%$ on h-BN). Planar isotropic strain does not qualitatively change the heat conduction in the perpendicular direction.

${ }^{27}$ K. Sun, M. A. Stroscio, and M. Dutta, Superlattices Microstruct. 45, 60 (2009).

${ }^{28}$ Z. Wei, Z. Ni, K. Bi, M. Chen, and Y. Chen, Phys. Lett. A 375, 1195 (2011).

${ }^{29}$ S. Baroni, P. Giannozzi, and E. Molinari, Phys. Rev. B 41, 3870 (1990).

${ }^{30}$ P. E. Hopkins, M. Baraket, E. V. Barnat, T. E. Beechem, S. P. Kearney, J. C. Duda, J. T. Robinson, and S. G. Walton, Nano Lett. 12, 590 (2012). 